\section{Multiple Sklerose}

Im Krankheitsverlauf erfahren etwa die Hälfte aller MS-Betroffenen eine Beeinträchtigung der kognitiven Fähigkeiten. Um dem entgegenzuwirken hat Teva in Zusammenarbeit mit dem Diplom-Psychologen Walter Kaiser und dem Computerspiele-Hersteller Chimera Entertainment („Gehirntraining mit Dr. Kawashima“) speziell auf die Bedürfnisse von MS-Erkrankten abgestimmte Übungen entwickelt, die kostenfrei auf www.aktiv-mit-ms.de zur Verfügung stehen.

Teva

\section{CIDP}

Bei Patienten mit Chronisch Inflammatorischer Demyelinisierender Polyneuropathie (CIDP) kann die Behandlung mit dem $10 \%$ igen i.v. Immunglobulin (IVIG) Privigen ${ }^{\circledR}$ zu einer signifikanten Verbesserung führen. Dies ist ein Ergebnis der PRIMA-Studie, die auf dem Meeting des Peripheral Nerve Society Inflammatory Neuropathy Consortium in Rotterdam vorgestellt wurde. In die offene Multicenter-Studie wurden sowohl mit IVIG vorbehandelte $(n=13)$ als auch nicht vorbehandelte $(n=15)$ CIDP-Patienten eingeschlossen. Sie erhielten eine Startdosis von $2 \mathrm{~g} / \mathrm{kg} \mathrm{KG} \mathrm{Privigen}{ }^{\circledR}$. Anschließend wurde über 22 Wochen in dreiwöchigen Intervallen je $1 \mathrm{~g} / \mathrm{kg}$ KG verabreicht. Primärer Endpunkt war die Responder-Rate gemäß INCATScore. Diese Rate betrug für die $\mathrm{Ge}$ samtstudienpopulation $60,7 \%$ und war signifikant höher als das vorher definierte Ziel von $35 \%$ (abgeleitet aus einem historischen Kollektiv von Placebo-behandelten Patienten). Der INCAT besserte sich im Verlauf signifikant von 3,7 auf 2,3. Die Patienten sprachen teils erst mit einer Latenz von mehreren Wochen auf die Therapie an, insbesondere jene, die im Rahmen der Studie erstmals IVIG erhielten. Es kann sinnvoll sein, erst nach längerer Gabe zu entscheiden, ob die CIDP auf die Behandlung anspricht. Der Hersteller hat den Antrag auf Zulassungserweiterung für CIDP eingereicht. red

CSL Behring

Nicht-kompetitiver AMPA-Rezeptorantagonist

\title{
Antiepileptikum mit neuem Wirkansatz
}

Mitte September 2012 wurde ein neues Antiepileptikum für die Zusatztherapie von Patienten mit fokalen Anfällen - mit und ohne sekundäre Generalisierung - in den Markt eingeführt: Perampanel zeichnet sich durch einen völlig neuen Wirkmechanismus aus: Es ist das erste antiglutamaterg wirkende Antiepileptikum.

Perampanel (Fycompa ${ }^{\circledR}$ ) ist ein selektiver, nicht-kompetitiver Antagonist von AMPA-Rezeptoren, die bei der Generierung und Ausbreitung von Anfällen im Gehirn eine wichtige Rolle spielen. Die Substanz verhindert die durch Glutamat induzierte Überstimulation der Rezeptoren auf postsynaptischen Neuronen und neutralisiert so einen Trigger bei der Auslösung fokaler Anfälle. „Da Perampanel nicht an der Glutamat-Bindungsstelle, sondern an einem nicht-kompetitiven Bindungsort des AMPA-Rezeptors andockt, wird es auch bei sehr hoher Glutamat-Konzentration im Hippocampus, die bei Epilepsiepatienten vor einem Anfall typisch sind, nicht vom Rezeptor verdrängt“, erläuterte Professor Martin Brodie, Universität Glasgow/Großbritannien.

\section{Hohe Responderrate}

Auf Basis von drei globalen Phase-IIIStudien wurde Perampanel im Sommer 2012 für die Zusatztherapie von Patienten ab zwölf Jahren mit fokal beginnenden Anfällen zugelassen. Sie umfassten knapp 1.500 Patienten, die trotz Therapie mit mindestens zwei Antikonvulsiva in den letzten zwei Jahren weiterhin an einfach oder komplex-fokalen Anfällen (mit oder ohne sekundäre Generalisierung) litten, berichtete Professor Bernhard Steinhoff, Epilepsiezentrum Kork, der das Studienkollektiv als „nicht einfach behandelbar" charakterisierte. Dennoch erwies sich Perampanel als hoch wirksam und führte ab der $4 \mathrm{mg}$-Dosis zu einer signifikanten Reduktion der Anfallsfrequenz und einem ebenfalls signifikanten Anstieg der Responderrate. Als bemerkenswert bezeichnete der Neurologe die Tatsache, dass insbesondere die schweren sekundär generalisierten klonisch-tonischen Anfälle stark reduziert wurden.

Langsames Aufdosieren empfohlen

Zudem kann Perampanel als insgesamt gut verträgliches Antikonvulsivum bezeichnet werden. Häufigste Nebenwirkungen sind Schwindel, Schläfrigkeit und Fatigue, die nach Steinhoffs Einschätzung im klinischen Alltag bei vorsichtiger Aufdosierung eher seltener auftreten werden als in den klinischen Studien. Er wies außerdem auf die lange Halbwertszeit der Substanz hin, die die tägliche Einmalgabe möglich mache.

Dr. Katharina Arnheim, freie Medizinjournalistin

\footnotetext{
European Media Launch Briefing „Fycompa ${ }^{\circledR}$ (Perampanel)" und Launch-Pressekonferenz „Perampanel (Fycompa $\left.{ }^{\circledR}\right)$ : Zulassung einer neuen Klasse von Antiepileptika" London, 12.9.2012; Veranstalter: Eisai
}

\section{Neue Immuntherapeutika erweitern das Behandlungsrepertoire}

In der Behandlung der Multiplen Sklerose stehen Erweiterungen der therapeutischen Möglichkeiten bevor. Neue Medikamente haben in Phase-III-Studien ihre therapeutische Wirksamkeit bei schubförmiger MS zeigen können.

Für die einmal tägliche orale Therapie der schubförmigen MS wurde kürzlich das krankheitsmodifizierende Teriflunomid (Aubagio ${ }^{\circledast}$ ), der aktive Hauptmetabolit von Leflunomid, von der US-amerika- nischen FDA zugelassen, berichtete Professor Bernd Kieseier von der Neurologischen Klinik der Heinrich-Heine-Universität Düsseldorf. Die Zulassung des Immunmodulators in Deutschland wird für Anfang nächsten Jahres erwartet.

Basis der Zulassung waren die Wirksamkeitsdaten der TEMSO-Studie [O' Connor et al. NEJM 2011; 365: $1293-$ 1303], die den Einfluss von Teriflunomid in zwei Dosierungen auf die Häufigkeit 